Tropical Journal of Pharmaceutical Research, December 2003; 2 (2): 215-221

(C) Pharmacotherapy Group

Faculty of Pharmacy, University of Benin

Benin City, Nigeria

All rights reserved.

Available online at http://www.tjpr.freehosting.net

Research Article

\title{
An alternative colorimetric method for the determination of chloramphenicol
}

\section{Chukwuenweniwe J Eboka ${ }^{\Phi}$, Johnson Smart and Sunday A Adelusi}

Faculty of Pharmacy, University of Benin, Benin City, Nigeria

\begin{abstract}
Purpose: To develop a simple, cheap, fast, accurate, sensitive and precise colorimetric method that can be used for the determination of chloramphenicol.

Method: Chloramphenicol was reduced in a mixture of glacial acetic acid and water using titanium (III) chloride at room temperature within $10 \mathrm{~min}$. The reduced product was then heated for 20 min with p-dimethylaminobenzaldehyde to yield the final product whose absorbance was used for the determination of the concentration of chloramphenicol. Results obtained with this method were compared with those obtained with the microbiological assay of chloramphenicol.
\end{abstract}

Result: The final product of the two step reaction was greenish - yellow in colour, absorbed strongly in the visible region and obeyed Beer's law at $\lambda_{\max }=440 \mathrm{~nm}$. The method developed was sensitive and accurately determined chloramphenicol in the presence of common excipients and in different dosage forms. There was statistically no significant difference ( $p<$ 0.05) between the results with the method developed and those obtained with the microbiological assay of chloramphenicol.

Conclusion: A simple, fast, cheap, precise, sensitive and accurate colorimetric method has been developed that could be routinely used for the determination of chloramphenicol in bulk drug and in different dosage forms. The advantage of the method is its speed and simplicity.

Key words: Chloramphenicol, colorimetric assay, microbiological assay, p-dimethylaminobenzaldehyde.

${ }^{\Phi}$ To whom correspondence should be addressed. 


\section{Introduction}

Chloramphenicol is one of the first widely used antibiotics. It was discovered in 1947 by Ehrlich et al amongst the metabolic products of Streptomyces venezuelae. The ability of chloramphenicol to inhibit rickettsiae in-vitro as well as a wide range of gram-positive and gram-negative bacteria gave rise to its immediate interest. This interest was further enhanced when the antibiotic was found to be well absorbed from the alimentary tracts of mice and $\operatorname{dog}^{1}$. It is bacteriostatic for most organisms but bacteriacidal to Haemophilus influencae.

Chloramphenicol acts by interfering with protein synthesis specifically by inhibiting the enzyme that transfers the peptide chain to the amino acid (puromycin) on the ribosome ${ }^{2}$. Recently, it has been shown that the drug causes single-strand breaks in Escherichia coli deoxyribonucleic acid ${ }^{3,4}$.

Chloramphenicol is commercially available in different dosage forms and can be administered as capsule, injection, eye drops and ear drops. Various methods are available for the assay of the compound in bulk drug, in dosage forms and in biological fluids ${ }^{5-20}$. These include colorimetric ${ }^{5-8}$, microbiological $^{9-11}$, radio-immunoassay ${ }^{12,} 13$, high performance liquid chromatography ${ }^{14-17}$, gas liquid chromatography ${ }^{18}$, spectrophotometric $^{19,}{ }^{20}$, and polarographic ${ }^{21}$ methods.

Although various colorimetric methods are available for the assay of the compound, they often lack speed and the experimental conditions are cumbersome and are thus prone to errors. The objective of the present work is to develop an alternative colorimetric method for the routine assay of chloramphenicol in bulk drug and dosage forms. This method is simple, fast, cheap, accurate and reproducible.

\section{Experimental}

\section{Materials}

All the reagents were of analytical grade and were used as received. Para-dimethylamino benzaldehyde and methanol were obtained from BDH Chemical Limited (Poole, United Kingdom). Titanium (III) chloride (14.5 $15.5 \%)$, glacial acetic acid and dimethyl sulphoxide were obtained from May and Baker (Dagenham, United Kingdom). Nutrient agar was obtained from Oxoid Limited (Bassingstoke, United Kingdom). Pure sample of chloramphenicol powder was obtained from Parke Davis Medical (East Leigh Hants, United Kingdom). Five different brands of chloramphenicol capsules, five brands of chloramphenicol injections, four brands of chloramphenicol eye drops and four brands of chloramphenicol ear drops were purchased from local pharmacies in Benin City, Nigeria.

The equipments used include a Spectronic D21 ultra - violet/visible spectrophotometer (Milton Roy Limited, USA), steam bath, shaker bath, hot box oven, autoclave and incubator (Gallenkamp, Birmingham, United Kingdom).

\section{Methods}

\section{Colorimetric method}

Pure chloramphenicol powder $(0.1 \mathrm{~g})$ was accurately weighed into a $20 \mathrm{ml}$ volumetric flask containing a mixture of $3 \mathrm{ml}$ each of glacial acetic acid and distilled water. The contents of the flask were shaken gently to dissolve the powder. Titanium (III) chloride $(3 \mathrm{ml})$ was then added to the flask and the contents mixed gently. The mixture was left to stand at room temperature for $10 \mathrm{~min}$ to achieve complete reduction of the nitro group of chloramphenicol to a primary aromatic amino group. The resulting solution $(1 \mathrm{ml})$ was then pipetted into a $25 \mathrm{ml}$ volumetric flask and made up to volume with methanol. From this solution, 0.2, 0.4, 0.6, 
0.8 and $1.0 \mathrm{ml}$ was each pipetted into different $25 \mathrm{ml}$ volumetric flasks. The content of each flask was made up to $1.0 \mathrm{ml}$ by adding $0.8,0.6,0.4,0.2$ and $0.0 \mathrm{ml}$ of methanol, respectively. Para-dimethylaminobenzaldehyde $(10 \mathrm{ml}$ of $0.1 \% \mathrm{w} / \mathrm{v}$ in methanol) was then added to each flask. The contents were mixed and heated on a steam bath for $20 \mathrm{~min}$. The flasks were brought out, allowed to cool to room temperature and each made up to volume with methanol. Blank was obtained by repeating the procedure but omitting the drug in the preparations. Time for the completion of each of the two reactions i.e. the reduction and the reaction with paradimethylaminobenzaldehyde were noted. The wavelength of maximum absorption $\left(\lambda_{\max }\right)$ of the product of reaction was also noted.

Stability of the final product was investigated by measuring the absorbance of the final product at $\lambda_{\max }$ at $0.5,1.0,2.0,3.0$ and $4.0 \mathrm{hr}$ after its formation. To test the sensitivity, reproducibility and limit of detection of the method developed, various standards of chloramphenicol were prepared. The concentrations of chloramphenicol in the final solutions of these standards ranged from 3.6 to $17.8 \mu \mathrm{g} / \mathrm{ml}$. The limit of detection was taken as twice the minimum concentration that could be determined reproducibly and falling in the linear portion of a regression line of a range of concentrations of the compound. To investigate the effects of starch and lactose, $0.2 \mathrm{~g}$ of each was separately mixed with 0.1 $\mathrm{g}$ of chloramphenicol. Each mixture was used in the place of the drug in the procedure above. For capsule and injection dosage forms, the weights of granules and powders respectively equivalent to $0.1 \mathrm{~g}$ of chloramphenicol were quickly weighed and used in the place of pure chloramphenicol in the procedure above. For chloramphenicol eye and ear drops, $2.0 \mathrm{ml}$ of each eye and ear drop was added to a mixture of $3.0 \mathrm{ml}$ glacial acetic acid and $1.0 \mathrm{ml}$ of distilled water. The procedure was then continued as for the pure drug above. A Beer's plot was made at the wavelength of maximum absorption from where the concentrations of unknowns were interpolated.

\section{Microbiological Method}

The method outlined in the British Pharmacopoeia $^{19}$ was adopted. Chloramphenicol solution $(5 \mathrm{mg} / \mathrm{ml})$ was made in dimethyl sulphoxide and diluted with distilled water to produce a $1 \mathrm{mg} / \mathrm{ml}$ solution. This solution was diluted serially with distilled water to produce different standard solutions containing $30,60,90$ and $120 \mu \mathrm{g} / \mathrm{ml}$ of chloramphenicol. An overnight broth culture of Sarcina lutea (ATOC 9341) obtained from the Department of Pharmaceutical Microbiology, University of Benin, Benin City was used in inoculating the surface of five nutrient agar plates. A sterile $4 \mathrm{~mm}$ cork borer was used to make wells in the agar plates. The standard chloramphenicol solutions $(0.4 \mathrm{ml}$ each) were placed into the wells. Dimethyl sulphoxide (without the drug) diluted serially as above was used as control and $0.4 \mathrm{ml}$ was also placed inside the well. The plates were maintained at room temperature for $30 \mathrm{~min}$ to allow diffusion of the drug into the medium and incubated at $37^{\circ} \mathrm{C}$ for $24 \mathrm{hr}$. The diameter of the zones of inhibition was then measured. Readings were done in duplicate and their averages taken.

For capsule and injection dosage forms, their granules and powders respectively were used to prepare solutions equivalent to 5 $\mathrm{mg} / \mathrm{ml}$ chloramphenicol in dimethyl sulphoxide. Thereafter, the procedure for the pure drug was followed. For the eye and ear drops, $1.0 \mathrm{ml}$ was taken and added to $9.0 \mathrm{ml}$ of dimethyl sulphoxide. This was mixed and diluted further with dimethyl sulphoxide to produce a solution equivalent to $5 \mathrm{mg} / \mathrm{ml}$ chloramphenicol. Each was then diluted serially with dimethyl sulphoxide to make different standard solutions and the procedure for the pure-drugs followed. A 
semi-logarithmic plot of logarithm of concentration versus average diameter of zone of inhibition was made from where concentrations of unknowns were interpolated.

\section{Analysis of data}

A statistical package called Instat (GraphPad Inc, USA) was used to compare the results obtained from the method developed and the microbiological assay of chloramphenicol for various dosage forms using 2-tailed paired Student $t$-test. At 95\% confidence interval, 2tailed $\mathrm{p}$ - values less than 0.05 were considered to be statistically significant.

\section{Results}

The procedure used in obtaining the final product involved two separate reactions. These are the initial reduction of the aromatic nitro group in chloramphenicol to a primary aromatic amine which in turn was reacted with p-dimethylaminobenzaldehyde. By withdrawing samples after different times of reduction, we established that the reduction of the nitro group in chloramphenicol using titanium (III) chloride under the experimental condition adopted here, reached completion in less than $10 \mathrm{~min}$. We also established that $10 \mathrm{ml}$ of $0.1 \%$ $\mathrm{w} / \mathrm{v}$ p-dimethylaminobenzaldehyde in methanol added to the reduction product and heated for 20 min was the optimum condition for the quantitative yield of the final product. The final product was greenish-yellow in colour and had a wavelength of maximum absorption $\left(\lambda_{\max }\right)$ of $440 \mathrm{~nm}$. At this wavelength, and under the experimental conditions followed here, Beer's law was obeyed for chloramphenicol reaction product. The regression equation of absorbance as a function of the concentration of chloramphenicol in the final product is $\mathrm{A}=0.0074+0.054 \mathrm{C}\left(\mathrm{r}^{2}=0.9995\right)$ where $\mathrm{A}$ is absorbance, $\mathrm{C}$ is concentration in $\mu \mathrm{g} / \mathrm{ml}$ and $\mathrm{r}$ is correlation coefficient. Limit of detection was found to be $1.05 \mu \mathrm{g} / \mathrm{ml}$. Coefficients of variation ranged from 0.61 to $2.17 \%$ at a concentration range of 1.05 $\mu \mathrm{g} / \mathrm{ml}$ to $17.8 \mu \mathrm{g} / \mathrm{ml}$. The excipients (lactose and starch) in the capsules did not interfere with the assay of chloramphenicol using this method developed. Recoveries of chloramphenicol from lactose and starch were $98.8 \pm 0.6 \%$ and $97.9 \pm 0.1 \%$, respectively.

Results of the assay of chloramphenicol in the different dosage forms using both the method developed and microbiological method are shown in the Table. We found no statistical difference between the method

Table: Percentage of label claim of chloramphenicol in different dosage forms \pm Standard error of the average

\begin{tabular}{cccc}
\hline $\begin{array}{c}\text { Dosage } \\
\text { form }\end{array}$ & $\begin{array}{c}\text { Method } \\
\text { Developed }\end{array}$ & $\begin{array}{c}\text { Microbiologic } \\
\text { al Method }\end{array}$ & $\begin{array}{c}\text { p-value } \\
\text { (2-tailed) }\end{array}$ \\
\hline Capsule & & & \\
A & $99.69 \pm 1.05$ & $101.66 \pm 0.68$ & 0.1656 \\
B & $11.42 \pm 0.18$ & $11.84 \pm 0.34$ & 0.2780 \\
C & $99.71 \pm 1.04$ & $101.93 \pm 0.83$ & 0.1445 \\
D & $98.98 \pm 0.95$ & $101.06 \pm 0.96$ & 0.1725 \\
E & $98.70 \pm 0.96$ & $100.28 \pm 0.74$ & 0.2376 \\
& & & \\
Injection & & & \\
A & $99.58 \pm 1.08$ & $99.83 \pm 1.26$ & 0.8847 \\
B & $98.83 \pm 0.24$ & $100.28 \pm 1.21$ & 0.2843 \\
C & $98.80 \pm 0.90$ & $101.38 \pm 0.83$ & 0.0790 \\
D & $98.98 \pm 0.95$ & $10105 \pm 0.96$ & 0.1761 \\
E & $99.51 \pm 0.62$ & $100.83 \pm 0.83$ & 0.2469 \\
& & & \\
Eye & & & \\
drop & $99.96 \pm 0.90$ & $99.88 \pm 1.13$ & 0.9574 \\
A & $99.27 \pm 0.87$ & $100.82 \pm 1.23$ & 0.3424 \\
B & $99.42 \pm 0.89$ & $100.49 \pm 0.74$ & 0.3882 \\
C & $98.41 \pm 0.53$ & $101.18 \pm 1.08$ & 0.0605 \\
D & & & \\
& & & \\
Ear & & & \\
drop & & & \\
A & $99.58 \pm 0.71$ & $100.90 \pm 1.03$ & 0.3295 \\
B & $99.40 \pm 0.80$ & $100.53 \pm 1.18$ & 0.4573 \\
C & $98.41 \pm 0.97$ & $100.49 \pm 0.74$ & 0.1380 \\
D & $99.90 \pm 0.49$ & $100.53 \pm 1.18$ & 0.6393 \\
& & & \\
\hline & & &
\end{tabular}


developed and the microbiological assay ( $p$ $<0.05$ ).

\section{Discussion}

We have developed an alternative colorimetric method in this study for the determination of chloramphenicol. This method is a modification of the method of Glazko and Wolf ${ }^{5}$ which involves the reduction of the nitro group in chloramphenicol with potassium borohydride in alkaline medium containing palladium and reacting the primary aromatic amine produced with $\mathrm{p}$-dimethylamino benzaldehyde to give a coloured product. The method of Glazko and Wolf ${ }^{5}$ is associated with problems including (1) the reducing agent is not very soluble in the medium used necessitating filtration to be carried out after the reduction process, and (2) the reaction is not quantitative. Titanium (III) chloride in glacial acetic acid chosen as the reducing agent of the nitro group of chloramphenicol in this study has been known to reduce aromatic and heter-aromatic nitro groups easily and quantitatively at room temperature ${ }^{22}$. This reduction process is shown in Scheme I.

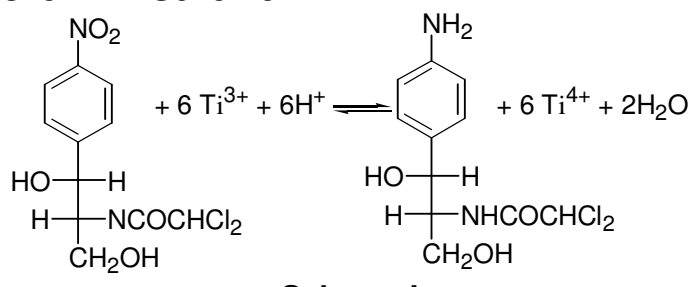

Scheme I

To ensure that the reaction proceeded favourably to the right, the amount of water must be minimised. The initial medium of equal volumes of glacial acetic acid : water $(1: 1 \mathrm{v} / \mathrm{v})$ has been established to be the optimum condition for aromatic nitro group reduction $^{22}$. Thus by simply mixing chloramphenicol and titanium (III) chloride in the solvent system, the reduction took place easily at room temperature within $10 \mathrm{~min}$. Prolonged reaction time at high temperature was avoided for the reduction. Colour development was achieved with $\mathrm{p}$ - dimethylaminobenzaldehyde as it reacted with the reduced chloramphenicol to form a coloured compound as shown in Scheme II.

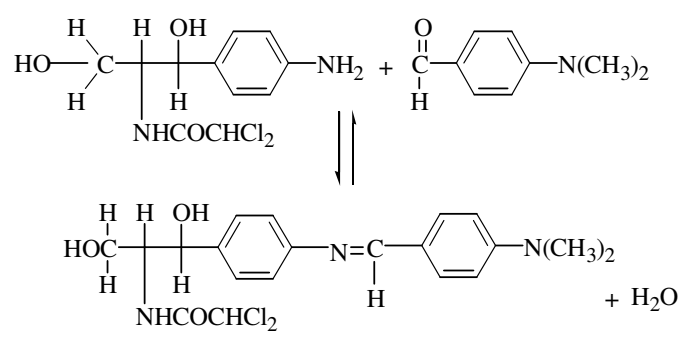

Scheme II

The reaction product contained an extensive conjugated double bond (chromophore) that absorbed in the visible region of the electromagnetic spectrum $\left(\lambda_{\max }=440 \mathrm{~nm}\right)$. Water is also a product of this reaction and should be minimised for product formation to proceed quantitatively. Use of methanol at all stages of the reaction minimised the amount of available water and also ensured that the products of the reactions dissolved easily.

Under the experimental conditions adopted, Beer's law was obeyed for different concentrations of chloramphenicol; thus each stage of the reaction was quantitative. At the wavelength of maximum absorption of the reaction product, $1.05 \mu \mathrm{g} / \mathrm{ml}$ of chloramphenicol gave an absorbance value of 0.05 . Thus, the method is very sensitive. Also, the final product was stable for over 4 hr. The reactions took place homogeneously, and can be easily carried out. Of particular note is the reduction reaction which took place quickly at room temperature.

When applied to assay chloramphenicol in different dosage forms, the method developed was found to be very precise. Assay results of chloramphenicol in all the different dosage forms investigated (except one brand of chloramphenicol capsule) met the requirements of chloramphenicol in the different dosage forms in respect of the percentage label claim $^{19}$. Both the method developed and microbiological method proved that one of the products investigated 
was substandard with content of chloramphenicol being 11.4 and $11.8 \%$, respectively. At $95 \%$ confidence interval, no significant difference was found between the data obtained using the method developed and the microbiological method.

Chloramphenicol has been determined colorimetrically using vanillin for colour development of the reduced chloramphenocol $^{23}$. A rapid and simple enzymatic assay of chloramphenicol has been developed which combined the specificity of the enzyme chloramphenicol acetyltransferase with the convenience of colorimetric detection ${ }^{24}$. The assay of chloramphenicol using this method was linear over the concentration range of $1.5-$ $65 \mu \mathrm{g} / \mathrm{ml}$. The linear range of the method developed in this study falls within this range. Colorimetric detection after high pressure liquid chromatography has also been used to study the pharmacokinetics of chloramphenicol in cows after intramuscular application and to monitor the levels of chloramphenicol in the blood and milk of goats following oral administration ${ }^{25,26}$. The method developed here can thus facilitate the colorimetric detection of chloramphenicol in the various applications.

\section{Conclusion}

An alternative colorimetric method has been developed that is very fast, sensitive, reproducible, simple and cheap to carry out yet gave accurate results. The developed method can be used for the routine assay of chloramphenicol in bulk drug and in pharmaceutical dosage forms.

The colour development in the final product involved the reaction of p-dimethylaminobenzaldehyde and the primary aromatic amine of reduced chloramphenicol. Any trace of primary aromatic amine in the reaction system will thus interfere in the colour development and hence affect the final result. In order to make this method specific for chloramphenicol, it is recommended that it should be extracted first or that any product that will yield any primary aromatic amine in the reaction system is removed.

\section{References}

1. Ehrlich J, Bartz QR, Smith RM, Joselyn AA. Chloramphenicol a New Antibiotic from Soil Actinomycetes. Science. 1947; 106: 417.

2. Rang HP and Dale MM. Pharmacology Second Edition. New York: Churchill Livingstone. $1991,819$.

3. Jackson CV. Antimicrobial Properties of Related Drugs. Pharm Sci. 1977; 28: 297.

4. David E. Antimicrobial Drug Action. New York: John Wiley and Sons, 1980, pp 216-8.

5. Glazko AJ, Wolf LM, Dill WA. A colorimetric method for the determination of chloramphenicol: Application to biochemical studies. J Clin Invest. 1949; 28: 940.

6. Shah RC, Raman PV, Sheth PV. Determination of chloramphenicol in blood. Indian $\mathrm{J}$ Pharm. 1968; 30: 68-9.

7. Pesez M and Bartos J. Diazo Reagent. 3 - Phenyl - 5 - nitroso-amino 1, 2, 4 - thiadiazole and 2 - Naphthol. Bull Soc Clin Fr. 1959; 1996.

8. Bartz QR. Isolation and characterization of Chloramphenicol. J Biol Chem 1948; 172" 445.

9. Joselyn DA, Ehrlich J, Schwab JL. Chloramphenicol assay and susceptibility test method. J Clin Invest. 1949; 28: 1051.

10. Randall WA, Kirstibaum A, Nielsen JK, Wintermore D. Diffusion-plate assay for chloramphenicol and auromycin. J Clin Invest. 1949; 28: 940.

11. Clarke EGC. Isolation and Identification of Drugs Vol. 2. London, UK: The Pharmaceutical Press, 1986, pp 221-5.

12. Davis AS, Davey MR, Clothier RC, Cocking EC. Quantification and comparison of chloramphenicol acetyl transferase activity in transformed plant protoplast using high performance liquid chromatography and radioisotopic base assays. Anal Biochem. 1992; 201(1): 87-91.

13. Nilsson-Ehle J, Nilsson-Ehle P. Assay of chloramphenicol in serum and cerebrospinal fluid by high performance liquid chromatography. Dis Clin Chem. 1983; 221-5.

14. Anhalt JP. Assay of gentamacin in serum and application to chloramphenicol by high performance liquid chromatography. Antimicrob Agents Chemother. 1977; 11: 6515.

15. Wong SH. Advances in chromatography for clinical drug analysis: supercritical fluid chromatography, capillary electrophoresis and selected high performance liquid chromatographic techniques. Ther Drug Monit. 1993; 15(6): 576-80. 
16. Aytron J, Evans MB, Harris AJ, Plumb RS. Porous graphic carbon shows promise for the rapid chromatographic analysis for polar drug metabolites. J Chromatogr B, Biomed Appl. 1995; 667(1): 173-8.

17. Nilsson - Ehle J, Yoshikawa TT, Scholz MC, Huze LB. Quantification of antibiotic using high performance liquid chromatography (HPLC). Antimicrob. Agents Chemother. 1976; 9: 75460.

18. Yamamoto M, Iguchi S, Aoyama T. Determination of chloramphenicol by gas-liquid chromatography. Chem Pharm Bull (Tokyo). 1967; 15: 123-7.

19. British Pharmacopoeia. London, Her Majesty's Stationery Office, 1988.

20. Schwarm EF, Dabner C, Wilson JW (Jr), Boghosian MP. Non-specificity of published assays for chloramphenicol J Pharm Sci. 1962; 55: 744-5.

21. Summa, AF. Polarographic determination of chloramphenicol preparations. J Pharm Sci. 1965; 54: 442-4.
22. Masonori S, Keiko K, Satomi J. Titanium (III) Chloride for the reduction of hetero - aromatic and aromatic nitro - compound. Chem Pharm Bull. 1980; 28(8): 2515-8.

23. Ahmed $\mathrm{BH}$, Onah JO. Colorimetric determination of chloramphenicol and metronidazole in pharmaceutical formulations after schiff-base formation with vanillin and anisaldehyde. global J Pure Applied Sci. 9(3) (http://:www.inasp.info/ journals/gjpas/vol9no3.htm/ - Assessed 3/10/03).

24. Morris HC, Miller J, Campbell RS, Hammond PM, Berry DJ, Price O. A rapid, enzymatic method for the determination of chloramphenicol in serum. J Antimicrob Chemother. 1988; 22 (6): 935-44.

25. Tanner $U$, Wuethrich A. Pharmacokinetics of chloramphenicol in cows after intramuscular application. Vet Res Commun. 1985; 9 (1): 2534.

26. Roy BK, Banerjee NC, Pandey SN. Distribution of chloramphenicol in blood and milk after oral administration in goat. Ind J Pharmac. 1983; 14 (4): 289-92. 